Research Paper

\title{
Hemoglobin Alc Can Identify More Cardiovascular and Metabolic Risk Profile in OGTT-Negative Chinese Population
}

\author{
Guicheng Peng $3^{3 *}$, Maohuan Lin ${ }^{1,2^{*}}$, Kun Zhang1,2, Jie Chen ${ }^{4}$, Yifang Wang 3 , Yu Yang 5 , Jingfeng Wang ${ }^{1,2,}$, \\ Hui Huang $1,2,{ }^{1}$ \\ 1. Department of Cardiology, Sun Yat-sen Memorial Hospital of Sun Yat-sen University, Guangzhou, 510120 China \\ 2. Guangdong Province Key Laboratory of Arrhythmia and Electrophysiology, Guangzhou, 510120 China \\ 3. Department of Internal Medicine, Dongguan Hengli Hospital, Dongguan, 523460 China \\ 4. Radiotherapy Department, Sun Yat-sen Memorial Hospital of Sun Yat-sen University, Guangzhou, 510120 China \\ 5. Department of Clinical Laboratory, Dongguan Hengli Hospital, Dongguan, 523460 China \\ * Guicheng Peng and Maohuan Lin contributed equally to the development of this research study.
}

$\triangle$ Corresponding author: Hui Huang Email: huangh8@mail.sysu.edu.cn. Jingfeng Wang Email: drwjfsums@gmail.com. 107 West Yanjiang Road, Department of Cardiology, Sun Yat-sen Memorial Hospital of Sun Yat-sen University, Guangzhou, China, 510120. Tel \# 0086-20-81332475. Fax \# 0086-20-81332623

(c) Ivyspring International Publisher. This is an open-access article distributed under the terms of the Creative Commons License (http://creativecommons.org/ licenses/by-nc-nd/3.0/). Reproduction is permitted for personal, noncommercial use, provided that the article is in whole, unmodified, and properly cited.

Received: 2013.0I.18; Accepted: 2013.05.24; Published: 2013.06.19

\begin{abstract}
Objective: To investigate the significance of hemoglobin Alc (HbAlc) in cardiovascular and metabolic risk stratification among diabetes and non-diabetes in southern Chinese.

Methods: Indigenous adults (aged more than or equal to 35 years) without known diabetes were enrolled in the cross-sectional survey. According to oral glucose tolerance test (OGTT), participants were categorized into OGTT-negative group and OGTT-positive group. Cardiovascular and metabolic risk profile was compared between different $\mathrm{HbAlc}$ levels $(\geq 6.5 \% \mathrm{vs}$. $<6.5 \%$ ) in each group.

Results: The prevalence of OGTT-diagnosed diabetes was $6.45 \%$ (422/6540). In OGTT-negative group, subjects with $\mathrm{HbAlc} \geq 6.5 \%$ were older, had higher prevalence of coronary heart disease, current smoking, hypertension, obesity and abdominal obesity. They also had higher body weight, waist-hip ratio, body mass index, glucose levels (fasting plasma glucose, 2-hour plasma glucose and $\mathrm{HbAlc}$ ), and lipid levels (total cholesterol and low density lipoprotein cholesterol). In OGTT-positive group, patients with $\mathrm{HbAlc} \geq 6.5 \%$ identified less cardiovascular and metabolic risk file than that in OGTT-negative group.

Conclusions: Subjects with $\mathrm{HbAlc} \geq 6.5 \%$ have more unfavorable cardiovascular and metabolic risk profile than those with $\mathrm{HbAlc}<6.5 \%$, especially in OGTT-negative population. More attention should be paid to this subgroup in clinical practice.
\end{abstract}

Key words: Diabetes mellitus, diagnosis, hemoglobin A1c, oral glucose tolerance test, risk stratification

\section{INTRODUCTION}

Diabetes is becoming a global health problem. As estimated by International Diabetes Federation [1], there were 366 million people suffering from diabetes worldwide in 2011, and by 2030 this figure will rise to 552 million. Diabetes also caused 4.6 million deaths and at least 465 billion US dollars in healthcare 
expenditures (11\% of total healthcare expenditures in adults aged 20-79 years) in 2011 [1].

Despite the recent advocating of hemoglobin A1c $(\mathrm{HbA} 1 \mathrm{c}) \geq 6.5 \%$ as a diabetes diagnostic tool by several international diabetes associations [2,3], for decades, the diagnosis of diabetes is mainly based on fasting plasma glucose (FPG) and/or 2-hour plasma glucose (2h-PG) after 75-g glucose challenge. As stated in the new diabetes guideline [2], the assay of $\mathrm{HbA1c}$ needs specially standardized and certified method. This technological-demanding will offset the high stability of HbA1c assay and will hinder its widespread use. On the other hand, the practical application of $\mathrm{HbA1c}$ as a diagnostic tool needs extensively verification in different countries with local diabetes epidemiological data, so does the determination of its optimal cut-off value. As a result, the introduction of $\mathrm{HbA} 1 \mathrm{c}$ into diabetes diagnostic protocol demands a certain period of time, especially in developing countries such as China, where data are limited.

There are strong reasons for considering diabetes as a risk equivalent of coronary heart disease (CHD) [4]. The presence of diabetes is often labeled as high or very high risk in contemporary clinical practice $[5,6]$. From this point of view, both $\mathrm{HbA1c}$ and oral glucose tolerance test (OGTT) are effective indicators for risk stratification. However, $\mathrm{HbA1c}$ and OGTT reflect different aspects of glucose metabolism. It raises the question whether $\mathrm{HbA1c}$ identifies different cardiovascular and metabolic risk profiles from OGTT. Several studies [7-10] have reported that diabetes diagnosed by $\mathrm{HbA1c}$ had higher body mass index (BMI), age, lipid levels or insulin resistance than those diagnosed by OGTT. To date, it remains unknown in Chinese diabetes population, the largest number in the world and manifesting postprandial hyperglycemia-oriented pattern [11], which is different from western counterparts [12]. Therefore, we designed the current survey to compare the difference of cardiovascular and metabolic risk profile between different $\mathrm{HbA1c}$ and glucose indicators category in a southern Chinese general population.

\section{METHODS}

\section{Study design and subjects}

Subjects were recruited from general population of 17 villages in southern China. The inclusion criteria were indigenous adult aged more than or equal to 35 years. People with previously-diagnosed diabetes (PDD), acute illness, anemia history, or incomplete data were excluded. Finally, 6,540 subjects were recruited. A flow chart was presented as Figure 1. All participants provided written informed consent to participate in the study.

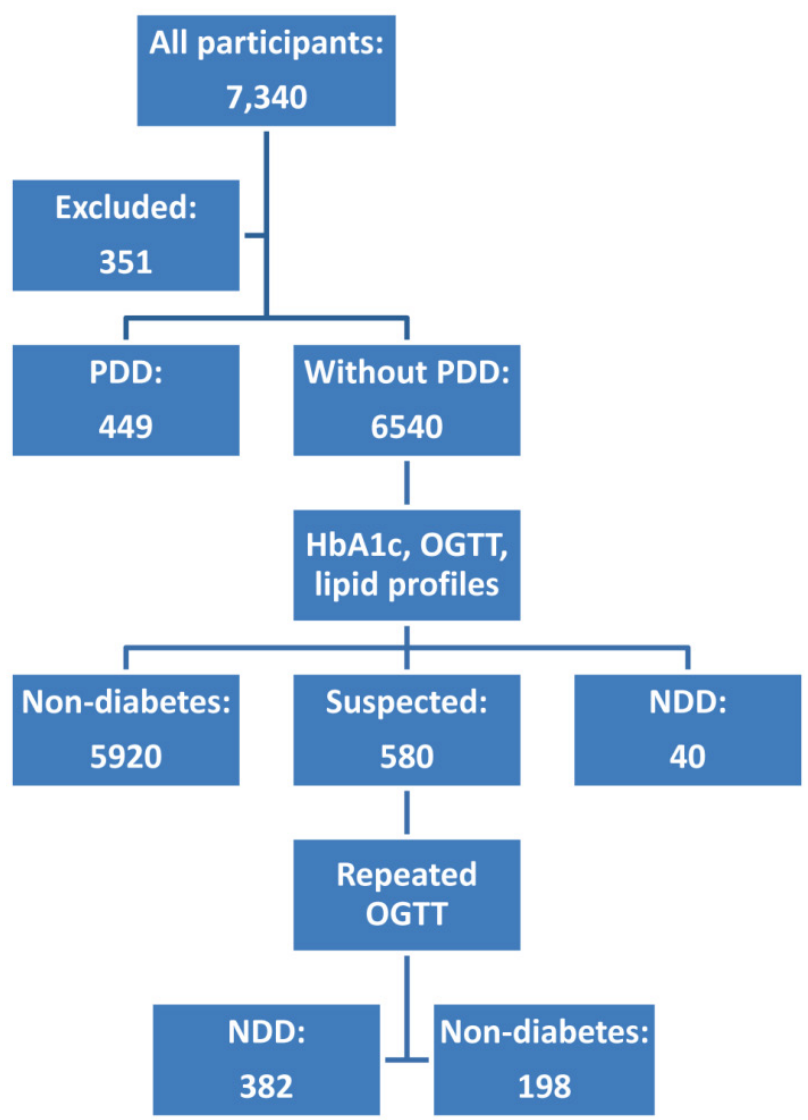

Figure I. Flow chart of trial. NDD, newly-detected diabetes; PDD, previously-diagnosed diabetes. All cases of diabetes were diagnosed according to OGTT results.

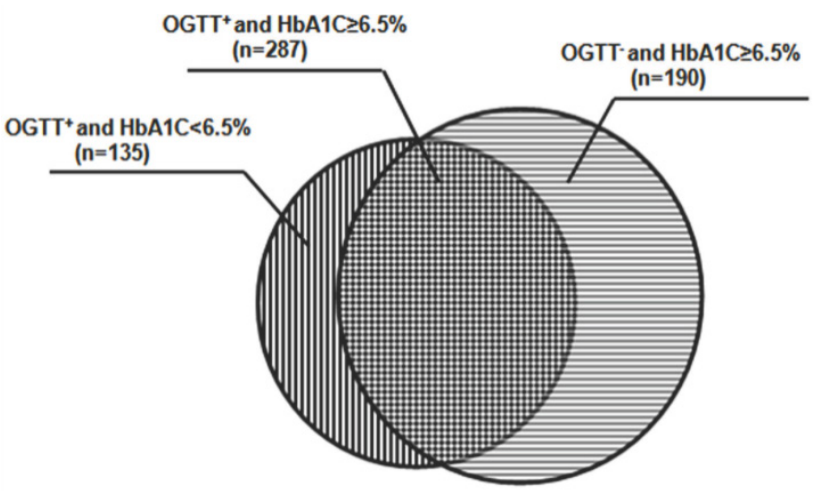

Figure 2. Number of the patients identified by OGTT and HbAIc test. 422 participants were diagnosed as diabetes according to OGTT. In these patients, 287 cases had their HbAlc level more than or equal to $6.5 \%$, and the remaining 135 patients had $\mathrm{HbAlc}$ level less than $6.5 \%$. In the participants diagnosed as non-diabetes according to OGTT, 190 cases had their HbAlc levels exceeded $6.5 \%$. 
The study complied with the declaration of Helsinki. The study protocol was approved by the Ethics Committee of Sun Yat-sen Memorial Hospital of Sun Yat-sen University.

\section{Questionnaire survey}

All participants completed a questionnaire, including items of demographic features, lifestyle, smoking and drinking habit, symptoms of hyperglycemia, family or personnel disease histories, especially CHD and related intervention (medicine and revascularization procedure), hypertension and antihypertensive drugs usage.

\section{Anthropometric measurements}

Anthropometric measurements were taken to obtain height, weight, waist circumference and hip circumference according to standard technique by trained investigators. Participants stood with bare feet close together, arms at the side and wore little clothing. Waist circumference was measured at the midpoint between the lower margin of the last rib and the top of the iliac crest. Hip circumference was measured around the widest portion of the buttocks, with the tape paralleled to the floor. Each measurement should be repeated twice. If the measurements were within 1 $\mathrm{cm}$ of one another, the average would be calculated. Otherwise, another two measurements should be repeated. Waist-hip ratio (WHR) was the ratio of the circumference of the waist to that of the hip. BMI was calculated by the participants' body mass $(\mathrm{kg})$ divided by the square of height $\left(\mathrm{m}^{2}\right)$. According to Chinese diabetes guideline [13], obesity is defined as BMI $\geq$ $2428 \mathrm{~kg} / \mathrm{m}^{2}$ and abdominal obesity is defined as waist circumference $\geq 90 \mathrm{~cm}$ in male or $\geq 85 \mathrm{~cm}$ in female.

Heart rate (HR) and blood pressure were recorded in all participants after rest for at least 5 minutes. Blood pressure was measured in the right arm in the sitting position with a mercury sphygmomanometer and proper bladder. Systolic blood pressure (SBP) and diastolic blood pressure (DBP) were identified by phase I and V (disappearance) Korotkoff sounds. Two measurements for each individual were taken with an interval of at least 1 minute. If the first two readings were quite different $(\geq 5 \mathrm{mmHg})$, an additional measurement was performed. The mean of these measurements was calculated and recorded. Hypertension was diagnosed as SBP $\geq 140 \mathrm{mmHg}$ and or $\mathrm{DBP} \geq 90 \mathrm{mmHg}$ or on anti-hypertensive treatment.

\section{Laboratory assay}

All participants underwent FPG, 2h-PG, HbA1c, total cholesterol (TC), triglyceride, high density lipoprotein cholesterol (HDL-C) and low density lipoprotein cholesterol (LDL-C) measurement. The detection of FPG and lipid profile needed an overnight fasting. OGTT was carried out according to World Health Organization (WHO) standardized protocol [14]. Venous blood samples were collected into vacuum tubes containing ethylenediaminetetraacetic acid (EDTA). Plasma was collected and stored at $-80^{\circ} \mathrm{C}$ until future detection. HbA1c test was performed with whole blood specimen at the day of blood collection. While other tests were performed collectively with stored plasma. Internal quality control was carried out at low, medium and high level every day before HbA1c assay. All tests follow manufacturers' instructions. Room temperature and humidity were controlled at $15-20{ }^{\circ} \mathrm{C}$ and $50 \%-60 \%$, respectively.

$\mathrm{HbA1c}$ was assayed by ion-exchange high-performance liquid chromatography (D-10, Bio-Rad Laboratories, Hercules, CA, USA), which had been certified by National Glycohemoglobin Standardization Program (NGSP) and was traceable to the International Federation of Clinical Chemistry and Laboratory Medicine (IFCC) reference method. Plasma glucose (FPG and 2h-PG) were measured by glucose oxidase-perioxidase method with TBA-120 auto-analyzer (Toshiba Medical Systems, Japan). TC (cholesterol oxidase-perioxidase aminopyrine method), triglyceride (glycerol phosphate oxidase-perioxidase aminopyrine method), HDL-C (direct method) and LDL-C (direct method) were measured using TBA-120 auto-analyzer (Toshiba Medical Systems, Japan).

\section{Diabetes diagnosis}

Currently, $\mathrm{HbA1c}$ is not accepted as a criterion for diabetes in China. As a result, all cases of newly-detected diabetes (NDD) were diagnosed by standard OGTT criteria according to American Diabetes Association (ADA) guideline [2]: FPG $\geq$ $7.0 \mathrm{mmol} / 1(126 \mathrm{mg} / \mathrm{dl})$ or $2 \mathrm{~h}-\mathrm{PG} \geq 11.1 \mathrm{mmol} / 1$ (200 $\mathrm{mg} / \mathrm{dl}$ ) during an OGTT, in the absence of unequivocal hyperglycemia, these criteria should be confirmed by repeat testing.

\section{Statistical analysis}

All statistical analyses were processed with SPSS 13.0 statistical package (SPSS, Inc., Chicago, IL). Continuous variables were expressed as mean \pm standard deviation when in normal distribution, or median (P2.5-P97.5) in skewed distribution. Categorical variables were expressed as numbers and percentages. Receiver operating characteristic (ROC) curve was constructed to calculate sensitivity and specificity of $\mathrm{HbA} 1 \mathrm{c}$ at $6.5 \%$ for diabetes diagnosis, using OGTT as the gold standard. The difference between participants with $\mathrm{HbA1c} \geq 6.5 \%$ and $\mathrm{HbA1c}<6.5 \%$ in OGTT-negative group and OGTT-positive group was tested by t-test, nonparametric test, or Pearson 
Chi-square test according to the variable type and distribution. According to current guidelines [2, 4-6], blood pressure, plasma lipid and glucose levels were correlated with age, gender, current smoking and weight, as a result, analysis of covariance (ANCOVA) was used for correction when the differences of the latter were statistically significant. A two-tailed $P$ value $<0.05$ was considered to be statistically significant.

\section{RESULTS}

\section{General features}

A total of 6,540 participants were recruited, with mean age 51 (36-80) years, and $40.6 \%$ of them were male. 422 participants were diagnosed as diabetes according to OGTT. The prevalence of OGTT-diagnosed diabetes was $6.45 \%$. In these patients, 287 cases had their HbA1c level $\geq 6.5 \%$. In 6,118 OGTT-negative participants, 190 cases had their $\mathrm{HbA1c}$ levels exceeded 6.5\% (Figure 2). The sensitivity and specificity of $\mathrm{HbA} 1 \mathrm{c}$ at $6.5 \%$ were $66.8 \%$ and $97.1 \%$, respectively.

\section{Comparison between $\mathrm{HbAl} \mathrm{c} \geq \mathbf{6 . 5 \%}$ and HbAlc $<6.5 \%$ among OGTT-negative partic- ipants}

In contrast to subjects with $\mathrm{HbA} 1 \mathrm{c}<6.5 \%$, subjects with $\mathrm{HbA1c} \geq 6.5 \%$ were older, had higher prevalence of CHD, current smoking, hypertension, obesity and abdominal obesity. They also had higher body weight, WHR, BMI, TC, LDL-C, FPG, 2h-PG and $\mathrm{HbA1c}$ than that of subjects with HbA1c $<6.5 \%$. No significant difference was shown in gender, SBP, DBP, HR, TG and HDL-C. (Table 1)

\section{Comparison between $\mathrm{HbAlc} \geq \mathbf{6 . 5 \%}$ and HbAlc $<6.5 \%$ among OGTT-positive patients}

Compared with patients of $\mathrm{HbA1c}<6.5 \%$, patients with $\mathrm{HbA1c} \geq 6.5 \%$ had higher body weight, Waist circumference, WHR, and higher concentration of TC, triglyceride, FPG, 2h-PG and HbA1c. But they were younger, had lower prevalence of hypertension than those of $\mathrm{HbA} 1 \mathrm{c}<6.5 \%$. The differences between gender, smoking rate, SBP, DBP, HR, LDL-C and HDL-C levels, and the prevalence of CHD, obesity yielded no statistically significant (Table 2).

Table I. Comparison between $\mathrm{HbAlc} \geq 6.5 \%$ and $\mathrm{HbAlc}<6.5 \%$ in OGTT-negative group

\begin{tabular}{|c|c|c|c|}
\hline & $\begin{array}{l}\text { HbA1c }<6.5 \% \\
(n=5928)\end{array}$ & $\begin{array}{l}\text { HbA1c } \geq 6.5 \% \\
(n=190)\end{array}$ & $\mathrm{P}$ value \\
\hline Age, years & $50(36-79)$ & $59(40-83)$ & 0.000 \\
\hline Male, \% & $40.1(2376 / 5928)$ & $42.6(81 / 190)$ & 0.480 \\
\hline $\mathrm{CHD}, \%$ & $1.1(64 / 5928)$ & $3.2(6 / 190)$ & 0.008 \\
\hline Smoking, \% & $24.8(1472 / 5928)$ & $36.8(70 / 190)$ & 0.000 \\
\hline \multicolumn{4}{|l|}{ Anthropometric index } \\
\hline Weight, kg & $60(42-84)$ & $64(45-92)$ & 0.000 \\
\hline BMI, $\mathrm{kg} / \mathrm{m}^{2}$ & $24.2(18.3-32.0)$ & $26.6(18.5-33.7)$ & 0.000 \\
\hline $\mathrm{BMI} \geq 24 \mathrm{~kg} / \mathrm{m}^{2}, \%$ & $52.3(3091 / 5906)$ & $71.4(135 / 189)$ & 0.000 \\
\hline Waist circumference, $\mathrm{cm}$ & $81(65-100)$ & $87(71-106)$ & 0.000 \\
\hline Hip circumference, $\mathrm{cm}$ & $93(82-108)$ & $96(83-112)$ & 0.000 \\
\hline WHR & $0.87(0.75-1.00)$ & $0.90(0.78-1.05)$ & 0.000 \\
\hline Abdominal obesity, \% & $30.2(1769 / 5865)$ & $40.6(76 / 187)$ & 0.002 \\
\hline \multicolumn{4}{|l|}{ Hemodynamic index* } \\
\hline $\mathrm{SBP}, \mathrm{mmHg}$ & $128(102-174)$ & 135(107-186) & 0.897 \\
\hline $\mathrm{DBP}, \mathrm{mmHg}$ & $74(57-98)$ & $75(60-102)$ & 0.393 \\
\hline $\mathrm{HR}, \mathrm{bpm}$ & $80(61-108)$ & $81(60-106)$ & 0.999 \\
\hline Hypertension, \% & $24.7(1464 / 5917)$ & $36.5(69 / 189)$ & 0.000 \\
\hline \multicolumn{4}{|l|}{ Lipid index* } \\
\hline $\mathrm{TC}, \mathrm{mmol} / \mathrm{L}$ & $5.14(3.46-7.29)$ & $5.61(3.29-7.93)$ & 0.004 \\
\hline $\mathrm{TG}, \mathrm{mmol} / \mathrm{L}$ & $1.20(0.50-3.94)$ & $1.47(0.54-4.56)$ & 0.169 \\
\hline HDL-C, mmol/L & $1.43(0.90-2.33)$ & $1.40(0.85-2.07)$ & 0.240 \\
\hline LDL-C, mmol/L & $3.05(1.29-4.93)$ & $3.37(1.22-5.72)$ & 0.002 \\
\hline \multicolumn{4}{|l|}{ Glucose index* } \\
\hline $\mathrm{FPG}, \mathrm{mmol} / \mathrm{L}$ & $5.4(4.5-6.6)$ & 6.1(4.9-7.4) & 0.000 \\
\hline 2h-PG, mmol/L & $6.4(3.5-11.0)$ & $8.0(3.8-13.5)$ & 0.000 \\
\hline HbA1c, \% & $5.7(4.8-6.3)$ & $6.6(6.5-7.4)$ & 0.000 \\
\hline
\end{tabular}

BMI, body mass index; CHD, coronary heart disease; SBP, systolic blood pressure; DBP, diastolic blood pressure; FPG, fasting plasma glucose; HbA1c, hemoglobin A1c; HDL-C, high density lipoprotein cholesterol; HR, heart rate; LDL-C, low density lipoprotein cholesterol; SBP, systolic blood pressure; TC, total cholesterol; WHR, waist-hip ratio; 2h-PG, 2-hour plasma glucose. * adjusted by age, body weight, current smoking. 
Table 2. Comparison between $\mathrm{HbAlc} \geq 6.5 \%$ and $\mathrm{HbAlc}<6.5 \%$ in OGTT-positive group

\begin{tabular}{|c|c|c|c|}
\hline & $\begin{array}{l}\text { HbA1c }<6.5 \% \\
(n=135)\end{array}$ & $\begin{array}{l}\text { HbA1c } \geq 6.5 \% \\
(n=287)\end{array}$ & P value \\
\hline Age, years & $60(38-88)$ & $55(36-81)$ & 0.002 \\
\hline Male, \% & $43.0(58 / 135)$ & $48.8(140 / 287)$ & 0.264 \\
\hline $\mathrm{CHD}, \%$ & $3.7(5 / 135)$ & $1.7(5 / 287)$ & 0.217 \\
\hline Smoking, \% & $30.4(41 / 135)$ & $27.9(80 / 287)$ & 0.597 \\
\hline \multicolumn{4}{|l|}{ Anthropometric index } \\
\hline Weight, kg & $63.0(37.8-91.3)$ & $66(46-94)$ & 0.004 \\
\hline BMI, $\mathrm{kg} / \mathrm{m}^{2}$ & 26.1(18.4-33.8) & $26.8(20.8-33.9)$ & 0.078 \\
\hline $\mathrm{BMI} \geq 24 \mathrm{~kg} / \mathrm{m} 2, \%$ & $71.6(96 / 134)$ & $78.6(224 / 285)$ & 0.118 \\
\hline Waist circumference, $\mathrm{cm}$ & $87(67-107)$ & $89(72-108)$ & 0.003 \\
\hline Hip circumference, $\mathrm{cm}$ & $96(82-112)$ & $96(84-111)$ & 0.176 \\
\hline WHR & $0.90(0.73-1.03)$ & $0.93(0.81-1.04)$ & 0.003 \\
\hline Abdominal obesity, $\%$ & $36.6(49 / 134)$ & $46.6(131 / 281)$ & 0.053 \\
\hline \multicolumn{4}{|l|}{ Hemodynamic index* } \\
\hline $\mathrm{SBP}, \mathrm{mmHg}$ & 143(102-195) & 137(109-188) & 0.201 \\
\hline $\mathrm{DBP}, \mathrm{mmHg}$ & $81(58-104)$ & $80(62-104)$ & 0.702 \\
\hline $\mathrm{HR}, \mathrm{bpm}$ & $84(57-128)$ & $85(63-115)$ & 0.540 \\
\hline Hypertension, \% & $59.0(79 / 134)$ & $46.7(134 / 287)$ & 0.019 \\
\hline \multicolumn{4}{|l|}{ Lipid index* } \\
\hline $\mathrm{TC}, \mathrm{mmol} / \mathrm{L}$ & $5.28(3.07-7.95)$ & $5.63(3.44-8.04)$ & 0.005 \\
\hline $\mathrm{TG}, \mathrm{mmol} / \mathrm{L}$ & $1.64(0.65-5.27)$ & $1.95(0.72-10.70)$ & 0.002 \\
\hline HDL-C, mmol/L & $1.31(0.86-1.97)$ & $1.26(0.86-2.13)$ & 0.806 \\
\hline LDL-C, mmol/L & $3.16(1.37-5.68)$ & $3.32(1.15-5.30)$ & 0.626 \\
\hline \multicolumn{4}{|l|}{ Glucose index* } \\
\hline $\mathrm{FPG}, \mathrm{mmol} / \mathrm{L}$ & $6.8(4.9-9.2)$ & $8.5(6.2-20.6)$ & 0.000 \\
\hline 2h-PG, mmol/L & $12.2(6.6-18.2)$ & 17.7(7.2-34.7) & 0.000 \\
\hline HbA1c, \% & $6.0(5.1-6.4)$ & $7.7(6.5-13.3)$ & 0.000 \\
\hline
\end{tabular}

BMI, body mass index; CHD, coronary heart disease; DBP, diastolic blood pressure; FPG, fasting plasma glucose; HbA1c, hemoglobin A1c; HDL-C, high density lipoprotein cholesterol; HR, heart rate; LDL-C, low density lipoprotein cholesterol; SBP, systolic blood pressure; TC, total cholesterol; WHR, waist-hip ratio; 2h-PG, 2-hour plasma glucose. * adjusted by age, body weight, current smoking.

\section{DISCUSSION}

Our study demonstrated that, compared with participants with $\mathrm{HbA1c}<6.5 \%$, subjects with $\mathrm{HbA} 1 \mathrm{c}$ $\geq 6.5 \%$ had more unfavorable cardiovascular and metabolic risk profiles, especially in OGTT-negative participants. Our results suggested that $\mathrm{HbA1c}$ could further stratify cardiovascular and metabolic risk beyond OGTT. More attention should be paid to those with $\mathrm{HbA} 1 \mathrm{c} \geq 6.5 \%$ but normal OGTT result in clinical practice. It was noteworthy that the difference of SBP between $\mathrm{HbA} 1 \mathrm{c} \geq 6.5 \%$ and $\mathrm{HbA1c}<6.5 \%$ in two groups $(7 \mathrm{mmHg}$ and $6 \mathrm{mmHg}$, respectively) were not statistically significant after adjustment. However, as stated by JNC-7 [15], death from both ischemic heart disease and stroke increases progressively and linearly from BP levels 115/75 mmHg upward. Furthermore, a meta-analysis from Blood Pressure Lowering Treatment Trialists' Collaboration has demonstrated that $4-8 \mathrm{mmHg}$ change in mean SBP is associated with decrease of major adverse cardiovascular events (stroke, CHD, heart failure, cardiovascular death and total mortality) [16]. So, the differences of SBP in our population are considered to be clinically important, although not statistically significant.

$\mathrm{HbA1c}$ and the traditional glucose indicators
(FPG and 2h-PG) reflect different aspects of glucose metabolism, it is interesting to explore whether $\mathrm{HbA1c}$ identifies different cardiovascular and metabolic risk profiles from OGTT. Several studies have focused on the disparity between $\mathrm{HbA1c}$ and glucose indicators across several ethnic groups. But their studies had smaller scale than ours and were mostly post-hoc analysis. Boronat et al [7] researched into 964 individuals from Spain and reported that subjects with $\mathrm{HbA1c} \geq 6.5 \%$ had higher values of BMI, waist circumference, FPG, fibrinogen and homeostasis model assessment of insulin resistance (HOMA-IR), but lower values for HDL-C. Kim et al [8] analyzed data of 1,491 Koreans, who were categorized by FPG $(\geq 7.0 \mathrm{mmol} / \mathrm{L})$ and $\mathrm{HbA1c}(\geq 6.5 \%)$, and they found that HbA1c-diagnosed diabetes group had significantly older age, higher proportion of women, while FPG-diagnosed diabetes group had higher SBP or DBP, fasting serum insulin, and HOMA-IR. Another epidemiology survey [9] from South Korea reported that the discordance between the two diabetes criteria (HbA1c and FPG) was significantly associated with obesity and older age. The Guangdong Health Survey 2010 (GHS 2010) [10] involved 3,590 residents aged 18 years of age or above and found that, compared to diabetes diagnosed by glucose indicators, patients 
with $\mathrm{HbA} 1 \mathrm{c} \geq 6.5 \%$ were generally older, and had higher BMI, waist circumference, blood pressure, lipids (except for HDL-C) and insulin levels.

Our data also demonstrated that HbA1c identified more cardiovascular and metabolic risk than OGTT, especially in OGTT-negative group. In OGTT-negative group, subjects with $\mathrm{HbA} 1 \mathrm{c} \geq 6.5 \%$ were older, had higher prevalence of coronary heart disease, current smoking, hypertension, obesity and abdominal obesity. They also had higher body weight, waist-hip ratio, BMI, glucose levels (fasting plasma glucose, 2-hour plasma glucose and HbA1c), and lipid levels (total cholesterol and low density lipoprotein cholesterol). Our results are not exactly the same as other studies in risk profiles. Differences in ethnicity, dietary and hyperglycemia patterns, study population, study design and diagnostic criteria might be the reasons for the discrepancy of our data and the others. Several studies have demonstrated that $\mathrm{HbA1c}$ level may vary with patients' ethnicity that are independent of glucose level $[17,18]$. Bloomgarden et al [19] showed that almost $70 \%$ of the intra-individual hemoglobin glycation variation was assumed to be genetically pre-determined. Chinese feed on carbohydrate-enriched foods and demonstrate higher proportion of postprandial hyperglycemia compared with western people. As showed by national diabetes survey of China by Yang et al [11], $46.6 \%$ of the participants with newly-diagnosed diabetes had isolated high $2 \mathrm{~h}-\mathrm{PG}$ levels, comparing with only $29 \%$ in the European population [12]. Nevertheless, our data and the aforementioned studies demonstrate consistently that $\mathrm{HbA1c}$ identifies different cardiovascular and metabolic risk profile from FPG and 2h-PG.

Diabetes has been considered as a risk equivalent of CHD [4]. The presence of diabetes is often labeled as high or very high risk in contemporary clinical practice $[5,6]$. As a result, the capacity of $\mathrm{HbA} 1 \mathrm{c}$ to further identify high risk is reduced in this already high or very high risk population. More importantly, we expanded our research into OGTT-negative population and found that $\mathrm{HbA} 1 \mathrm{c} \geq 6.5 \%$ represented more unfavorable and significant cardiovascular and metabolic risk profile in this group. This finding underscores the feature of $\mathrm{HbA1c}$ in risk stratification in OGTT-negative population. That is, HbA1c can identify population with high cardiovascular and metabolic risk at an earlier stage than FPG and 2h-PG. As what has been proved by many surveys and clinical trials, the most benefit in diabetes management comes from early diagnosis and early treatment so as to prevent or delay the onset of costly and harmful complications. From this point of view, HbA1c may be a more suitable and practical diagnostic criterion for diabetes than FPG and 2h-PG.

The mechanism for HbA1c's better performance in risk stratification is not fully elucidated. HbA1c represents the average glucose level in the preceding 8-12 weeks. Therefore, $\mathrm{HbA1c}$ is a more stable indicator for glucose level in a certain period of time than FPG and 2h-PG. Several researches have reported that long-term poor glucose control is associated with endothelial dysfunction [20,21], arterial stiffness [22, 23], low-grade systemic inflammation [24], oxidative stress [25], and platelet activation [26]. However, the exact mechanism may be multi-factorial and interactive, because the above-mentioned pathophysiological changes often coexist and interact with each other.

In conclusion, subjects with $\mathrm{HbA} 1 \mathrm{c} \geq 6.5 \%$ have more unfavorable cardiovascular and metabolic risks than those with $\mathrm{HbA} 1 \mathrm{c}<6.5 \%$, especially in OGTT-negative population. This has important clinical implication because it indicates that this subgroup (subjects with $\mathrm{HbA} 1 \mathrm{c} \geq 6.5 \%$ and normal OGTT) may not be at low risk as previously thought, and more intensive management should be employed. Further studies are required for the mechanism of the discrepancy of $\mathrm{HbA} 1 \mathrm{c}$ in risk stratification of OGTT-negative and OGTT-positive population.

\section{Abbreviations}

BMI: body mass index; CHD: coronary heart disease; DBP: diastolic blood pressure; FPG: fasting plasma glucose; HbA1c: hemoglobin A1c; HDL-C: high density lipoprotein cholesterol; LDL-C: low density lipoprotein cholesterol; NDD, newly-detected diabetes; OGTT: oral glucose tolerance test; PDD, previously-diagnosed diabetes; ROC: receiver operating characteristic; SBP: systolic blood pressure; TC: total cholesterol; TG: triglyceride; 2h-PG: 2-hour plasma glucose; WHR: Waist-hip ratio.

\section{Acknowledgement}

This work was supported in part by National Natural Science Foundation of China [81170647, 91029742 and 30973207 to Hui Huang, 30971262 to Jingfeng Wang].

\section{Competing Interests}

The authors have declared that no competing interest exists.

\section{References}

1. [Internet] International Diabetes Federation. The Global Burden. http://www.idf.org/diabetesatlas/5e/the-global-burden. Accessed 24 Jun 2012 
2. American Diabetes Association. Standards of medical care in diabetes--2012. Diabetes care. 2012; 35 (Suppl 1):S11- S63.

3. The International Expert Committee. International Expert Committee report on the role of the $\mathrm{A} 1 \mathrm{C}$ assay in the diagnosis of diabetes. Diabetes care. 2009; 32:1327-1334.

4. Expert Panel on Detection, Evaluation, and Treatment of High Blood Cholesterol in Adults. Executive Summary of The Third Report of The National Cholesterol Education Program (NCEP) Expert Panel on Detection, Evaluation, And Treatment of High Blood Cholesterol In Adults (Adult Treatment Panel III). JAMA.2001; 285: 2486-2497.

5. Mancia G, De Backer G, Dominiczak A, et al. 2007 Guidelines for the management of arterial hypertension: The Task Force for the Management of Arterial Hypertension of the European Society of Hypertension (ESH) and of the European Society of Cardiology (ESC). Eur Heart J. 2007; 28:1462-1536.

6. Reiner Z, Catapano AL, De Backer G, et al. ESC/EAS Guidelines for the management of dyslipidaemias: the Task Force for the management of dyslipidaemias of the European Society of Cardiology (ESC) and the European Atherosclerosis Society (EAS). Eur Heart J. 2011; 32:1769-1818.

7. Boronat M, Saavedra P, Lopez-Rios L, et al. Differences in cardiovascular risk profile of diabetic subjects discordantly classified by diagnostic criteria based on glycated hemoglobin and oral glucose tolerance test. Diabetes care. 2010; 33: 2671-2673.

8. Kim $\mathrm{CH}$, Kim HK, Bae SJ, et al. Discordance between fasting glucose-based and hemoglobin A1c-based diagnosis of diabetes mellitus in Koreans. Diabetes Res Clin Pract. 2011; 91:e8-e10.

9. Kim JH, Shin JH, Lee HJ, et al. Discordance between HbA1c and fasting plasma glucose criteria for diabetes screening is associated with obesity and old age in Korean individuals. Diabetes Res Clin Pract. 2011; 94:e27-29.

10. Zhang $\mathrm{YH}, \mathrm{Ma}$ WJ, Thomas GN, et al. Diabetes and pre-diabetes as determined by glycated haemoglobin a1c and glucose levels in a developing southern Chinese population. PloS one. 2012; 7:e37260.

11. Yang W, Lu J, Weng J, et al. Prevalence of diabetes among men and women in China. N Engl J MED. 2010; 362:1090-1101.

12. [No authors listed]. Will new diagnostic criteria for diabetes mellitus change phenotype of patients with diabetes? Reanalysis of European epidemiological data. BMJ. 1998; 317:371-375.

13. Chinese Diabetes Society. China guideline for type 2 diabetes. Beijing, China: Peking University Medical Press; 2011.

14. Alberti KG, Zimmet PZ. Definition, diagnosis and classification of diabetes mellitus and its complications. Part 1: diagnosis and classification of diabetes mellitus provisional report of a WHO consultation. Diabet Med. 1998; 15:539-53.

15. Chobanian A, Bakris G, Black H, et al. Seventh report of the Joint National Committee on Prevention, Detection, Evaluation, and Treatment of High Blood Pressure. Hypertension. 2003; 42:1206-52.

16. Turnbull F, Blood Pressure Lowering Treatment Trialists' Collaboration. Effects of different blood-pressure-lowering regimens on major cardiovascular events: results of prospectively-designed overviews of randomised trials. Lancet. 2003; 362:1527-35.

17. Herman WH, Cohen RM. Racial and ethnic differences in the relationship between $\mathrm{HbA1c}$ and blood glucose: implications for the diagnosis of diabetes. J Clin Endocrinol Metab. 2012; 97:1067-1072.

18. Selvin E, Steffes MW, Ballantyne CM, et al. Racial differences in glycemic markers: a cross-sectional analysis of community-based data. Ann Intern Med. 2011; 154:303-309.

19. Bloomgarden ZT, Inzucchi SE, Karnieli E, et al. The proposed terminology 'A(1c)-derived average glucose' is inherently imprecise and should not be adopted. Diabetologia. 2008; 51:1111-1114.

20. Ce GV, Rohde LE, da Silva AM, et al. Endothelial dysfunction is related to poor glycemic control in adolescents with type 1 diabetes under 5 years of disease: evidence of metabolic memory. J Clin Endocrinol Metab. 2011; 96:1493-1499.

21. Lorbeer R, Empen K, Dorr M, et al. Association between glycosylated haemoglobin $\mathrm{A}(1 \mathrm{c})$ and endothelial function in an adult non-diabetic population. Atherosclerosis. 2011; 217:358-363.

22. Chen $Y$, Huang $Y$, Li X, et al. Association of arterial stiffness with HbA1c in 1,000 type 2 diabetic patients with or without hypertension. Endocrine. 2009; 36:262-267.

23. Gunarathne A, Patel JV, Kausar S, et al. Glycemic status underlies increased arterial stiffness and impaired endothelial function in migrant South Asian stroke survivors compared to European Caucasians: pathophysiological insights from the West Birmingham Stroke Project. Stroke. 2009; 40:2298-2306.
24. Natali A, Toschi E, Baldeweg S, et al. Clustering of insulin resistance with vascular dysfunction and low-grade inflammation in type 2 diabetes. Diabetes. 2006; 55:1133-1140.

25. Zheng F, Lu W, Jia C, et al. Relationships between glucose excursion and the activation of oxidative stress in patients with newly diagnosed type 2 diabetes or impaired glucose regulation. Endocrine. 2010; 37:201-208.

26. Shah B, Sha D, Xie D, et al. The relationship between diabetes, metabolic syndrome, and platelet activity as measured by mean platelet volume: the National Health And Nutrition Examination Survey, 1999-2004. Diabetes care. 2012; 35:1074-1078. 